OPEN ACCESS

Edited by:

Sandip D. Kamath,

James Cook University, Australia

Reviewed by:

Aya C. Taki,

James Cook University, Australia

Lionel Hebbard,

James Cook University, Australia

${ }^{*}$ Correspondence:

Michal A. Rahat mrahat@netvision.net.il;

rahat_miki@clalit.org.il

Specialty section:

This article was submitted to Vaccines and Molecular Therapeutics,

a section of the journal

Frontiers in Immunology

Received: 21 August 2018 Accepted: 28 November 2018 Published: 10 December 2018

Citation:

Simanovich E, Brod V and Rahat MA (2018) Active Vaccination With EMMPRIN-Derived Multiple Antigenic

Peptide (161-MAP) Reduces Angiogenesis in a Dextran Sodium Sulfate (DSS)-Induced Colitis Model.

Front. Immunol. 9:2919.

doi: 10.3389/fimmu.2018.02919

\section{Active Vaccination With EMMPRIN-Derived Multiple Antigenic Peptide (161-MAP) Reduces Angiogenesis in a Dextran Sodium
Sulfate (DSS)-Induced Colitis Model}

\author{
Elina Simanovich ${ }^{1}$, Vera Brod ${ }^{1}$ and Michal A. Rahat ${ }^{1,2 *}$ \\ ${ }^{1}$ Immunotherapy Laboratory, Carmel Medical Center, Haifa, Israel, ${ }^{2}$ The Ruth and Bruce Rappaport Faculty of Medicine, \\ Technion-Israel Institute of Technology, Haifa, Israel
}

Ulcerative colitis (UC) is an autoimmune disease that affects the colon and shares many clinical and histological features with the dextran sulfate sodium (DSS)-induced colitis model in mice. Angiogenesis is a critical component in many autoimmune diseases, as well as in the DSS-induced colitis model, and is it partially mediated by EMMPRIN, a multifunctional protein that can induce the expression of both the potent pro-angiogenic vascular endothelial growth factor (VEGF) and matrix metalloproteinases (MMPs). We asked whether targeting EMMPRIN by active vaccination, using a novel, specific epitope in the protein, synthesized as a multiple antigenic peptide (MAP), could trigger beneficial effects in the DSS-induced colitic C57BL/6J mice. Mice were vaccinated with four boost injections (50 $\mu \mathrm{g}$ each) of either 161-MAP coding for the EMMPRIN epitope or the scrambled control peptide (Scr-MAP) emulsified in Freund's adjuvant. We show that male mice that were vaccinated with 161-MAP lost less weight, demonstrated improved disease activity indices (DAl), had reduced colitis histological score, and their colons were longer in comparison to mice vaccinated with the Scr-MAP. The 161-MAP vaccination also reduced serum and colon levels of EMMPRIN, colon concentrations of VEGF, MMP-9, and TGF $\beta$, and vessel density assessed by CD31 staining. A similar effect was observed in female mice vaccinated with 161-MAP, including weight loss, colitis histological score, colon length, colon levels of EMMPRIN and colon concentrations of VEGF. However, for female mice, the changes in DAI values, EMMPRIN serum levels, and MMP-9 and TGF $\beta$ colon concentrations did not reach significance. We conclude that our strategy of alleviating autoimmunity in this model through targeting angiogenesis by actively vaccinating against EMMPRIN was successful and efficient in reducing angiogenesis.

Keywords: angiogenesis, multiple antigenic peptide (MAP), active peptide vaccination, DSS-induced colitis, EMMPRIN/CD147 


\section{INTRODUCTION}

Inflammatory bowel disease (IBD) is a group of chronic inflammatory diseases, with the two major diseases being Crohn's disease (CD) and ulcerative colitis (UC). UC is believed to be an autoimmune disease that primarily affects the large intestine, with unknown etiology. However, a local increase in the concentrations of reactive oxygen species (ROS) and proinflammatory cytokines (primarily, TNF $\alpha$, IL-1 $\beta$, and IL-17), was identified in UC patients, and these were speculated to increase the risk of colorectal cancer in chronic inflammation $(1-3)$.

The DSS-induced colitis model used in rats and mice is widely used as an experimental model of IBD that demonstrates clinical and histopathological features similar to the human autoimmune $\mathrm{UC}(3,4)$. DSS is thought to induce a chemical injury in intestinal epithelial cells, which causes them to lose barrier functions and consequently exposes the Lamina Propria (LP) to antigens and intestinal bacteria that enhance inflammation (1). Different concentrations of DSS, usually ranging between 1 and 3\%, have been used to achieve moderate, mild or severe intestinal injury, varying time of healing and repair accordingly (5). Similar to UC, the pro-inflammatory response and increased reactive oxygen species (ROS) are implicated in the continued tissue damage caused in DSS-induced colitis (2).

Angiogenesis has been linked to chronic inflammation, and has been shown to be a critical component of the pathogenesis of DSS-induced colitis and is associated with disease severity, as it promotes leukocyte influx and supplies the necessary oxygen and nutrients to the inflamed tissue (6). VEGF is a known potent pro-angiogenic factor that links angiogenesis and inflammation by promoting endothelial cell proliferation, migration, tube formation and vascular permeability, as well as increasing neutrophil adhesion through the activation of NF- $\kappa \mathrm{B}$ and increased expression of adhesion molecules (7, 8). Matrix metalloproteinases (MMPs) can remodel the ECM to facilitate endothelial cell migration, release VEGF that is bound to the ECM, or conversely, degrade collagen XVIII to produce the anti-angiogenic factor endostatin (9). Thus, both VEGF and MMPs, particularly MMP-9, promote angiogenesis. Increased permeability, characteristic of angiogenic vessels, further contributes to UC progression, as it reduces barrier functions and allows interaction of lumen bacteria with LP immune cells (10).

EMMPRIN (also called CD147 or basigin) is a transmembranal protein with multiple functions. Depending on the protein it binds to, EMMPRIN can be involved in cell metabolism when it chaperones the monocarboxylate transporters MCT-1 and MCT-4, it can serve as a leukocyte chemoattractant when it binds to extracellular cyclophilin A/B, and it becomes an adhesion molecule when it binds to integrins and to E-selectin, to name just a few functions (11-13). However, its most familiar activity is mediated through homophilic interactions of membranal-soluble or membranal-membranal EMMPRIN molecules (14), which induce the expression of VEGF and several types of MMPs, rendering EMMPRIN an important pro-angiogenic factor (15-18).
We have recently identified a novel epitope in the EMMPRIN protein extracellular domain I, which is specifically responsible for the induction of both VEGF and MMP-9 (19). We have synthesized this epitope as an octa-branched multiple antigenic peptide (designated 161-MAP), and used it either in a therapeutic or a prophylactic manner to vaccinate mice that were implanted with the CT26 colon carcinoma tumors subcutaneously, or that were intravenously injected with this cell line to generate an experimental metastasis model. Vaccination against EMMPRIN resulted in the inhibition, and even regression of both tumors and metastases, partly through the reduction in vessel density, and through reduced expression of EMMPRIN, VEGF and MMP-9, cumulatively decreasing angiogenesis. In view of the importance of angiogenesis in colitis, we now ask whether the same vaccination against EMMPRIN could also affect chronic inflammation in a mouse DSS-induced colitis model.

\section{RESULTS}

\section{1-MAP Active Vaccination Ameliorates Disease Severity in DSS-Induced Colitis}

We have chosen to vaccinate the mice with Scr-MAP or 161MAP in a prophylactic manner, before the induction of colitis by DSS, to allow the adaptive immunity to prepare fully to the DSS challenge. Four days after the last vaccine injection the mice were supplied with DSS dissolved in their drinking water for 5 consecutive days, and 15 days after the introduction of DSS we euthanized the mice. The design of the experiment is shown (Figure 1A). DSS induced colonic damage that was manifested both by weight loss and by the disease activity index (DAI), which was calculated by factoring in weight loss, diarrhea, and occult blood or rectal bleeding. In the Scr-MAP vaccinated control mice, weight loss and DAI gradually increased and peaked around day 9 both in male and female mice. Following this peak, mice exhibited reduction in the change of weight and in the DAI (Figure 1B), suggesting that repair of the colonic damage had begun. A similar trend was observed for the weight loss of the161MAP vaccinated mice, but although the peak was evident on the same days as the control group, it was reduced in comparison (by 1.65 -fold, $p<0.01$ for the male mice, by 2.9-fold, $p<0.05$ for the female mice). Disease severity, assessed by the DAI, showed significant results only for male mice. It gradually elevated and peaked around day 9, and then moderately declined in both ScrMAP and 161-MAP vaccinated mice, but not back to the levels prior to DSS administration. However, in days 9 through 12, the disease in the control group was more severe (by 23 and 35\%, $p$ $<0.05$, Figure 1C). This observation suggests that damage and inflammation are still preset 10 days after mice were no longer exposed to DSS, and that the 161-MAP active vaccination exerted a protective influence. It is also noteworthy that starting on day 10 , the Scr-MAP vaccinated females demonstrated significantly reduced change in weight $(p<0.001)$ and DAI scores $(p<0.01)$, in comparison to the Scr-MAP vaccinated males, suggesting that the females exhibited reduced inflammation.

Histological analysis of the colon in the control groups revealed damage to the epithelial layer, crypt loss and destruction, 
A

\section{1-MAP/Scr-MAP \\ Vaccinations}

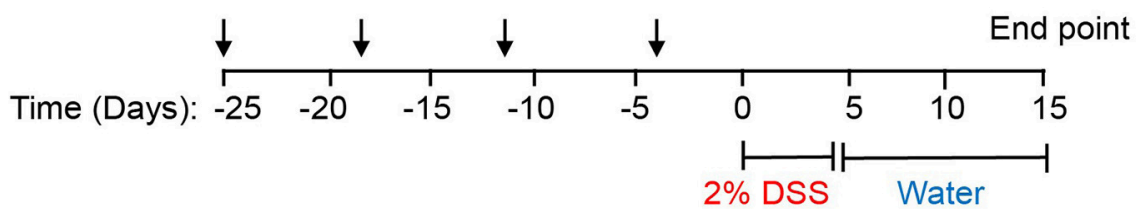

B Weight loss
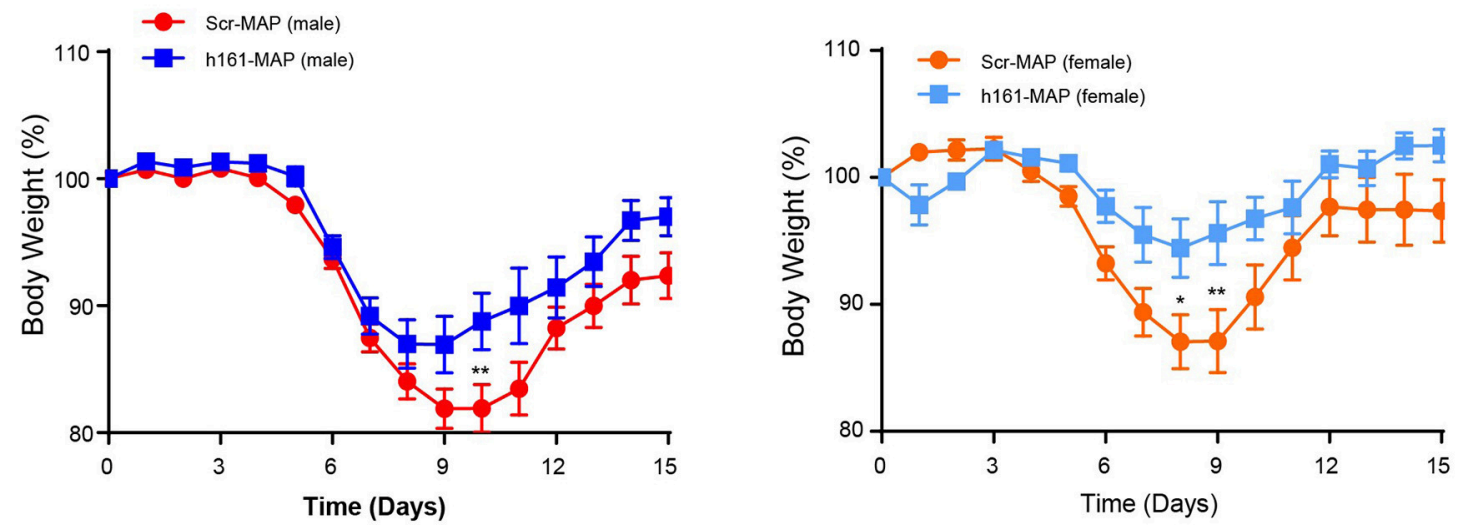

C Disease activity index (DAI)
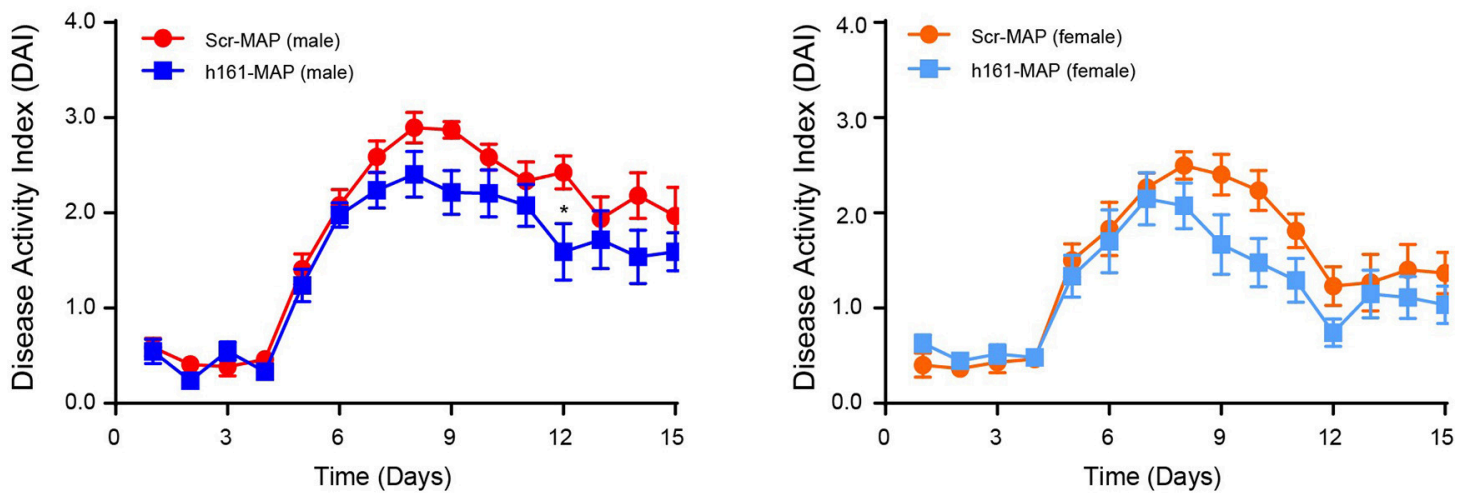

FIGURE 1 | 161-MAP active vaccination improves disease severity. (A) The experimental design is shown. Male and female C57BI/6J mice were randomly assigned to the Scr-MAP vaccinated control group, or to the 161-MAP vaccinated experimental group. Mice received four vaccine boost injections every 7 days (arrows) prior to administration of $2 \%$ DSS in their drinking water for 5 consecutive days (marked). For the remaining time the mice received regular water, and after 10 days they were euthanized. After receiving DSS, mice were monitored daily and their (B) loss of weight or $\mathbf{( C )}$ the disease activity index (DAI) were observed ( $n=12$ for male mice, $n=9$ for female mice). ${ }^{*} p<0.05,{ }^{* *} p<0.01$ relative to the Scr-MAP vaccinated mice at the same day.

and increased leukocyte infiltration to the lamina propria (LP) and to the submucosa (Figures 2A,B). In comparison, in the 161MAP vaccinated groups, crypt structures and epithelial lining were less damaged, and the immune infiltrate was reduced, as was reflected in the histological scores (Figure 2B, 95\% CI for males [1.6, 10.15], 95\% CI for females [0.1, 5.02]). Additionally, colon length, where shortening is a marker of inflammation, was increased in the 161-MAP vaccinated mice (by $12 \%$ in both male and female mice, $p<0.05,95 \%$ CI for males $[0.14,1.6]$, and for females $[0.4,1.6]$, Figures 2C,D).

\section{1-MAP Active Vaccination Reduces Angiogenesis}

To examine whether the 161-MAP active vaccination targeted the EMMPRIN protein in the colon, we stained for the protein in the colon tissue sections. EMMPRIN is highly expressed in the colon, 

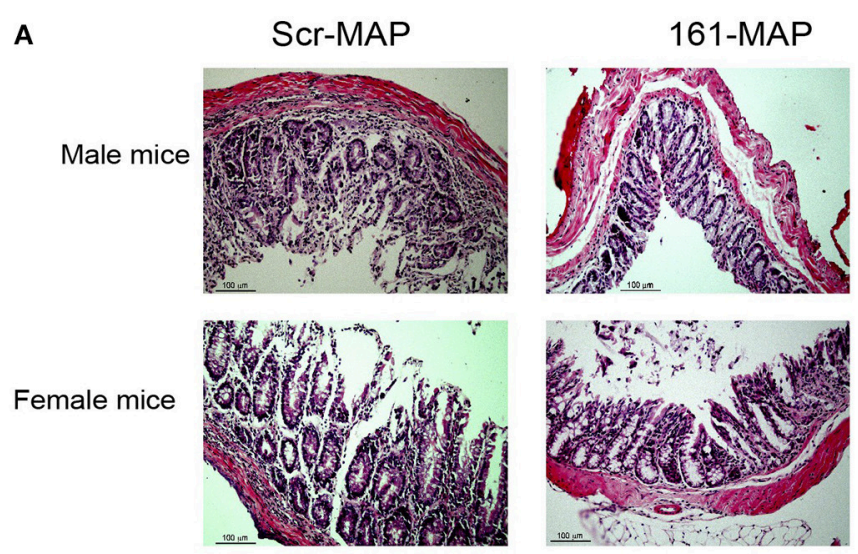

B
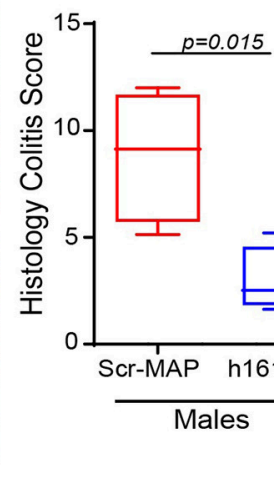

C

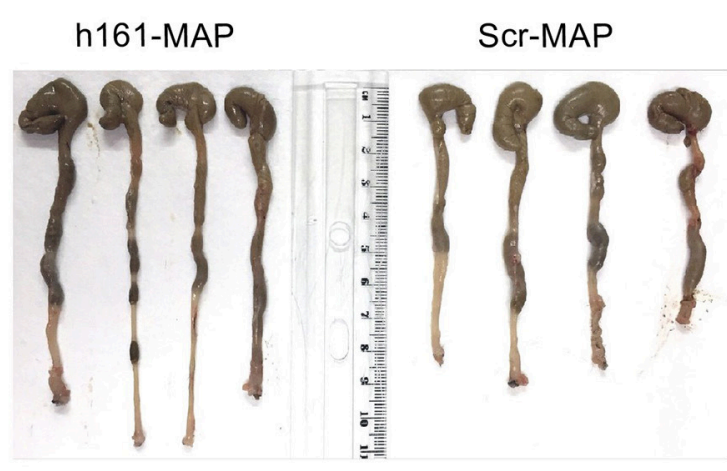

D

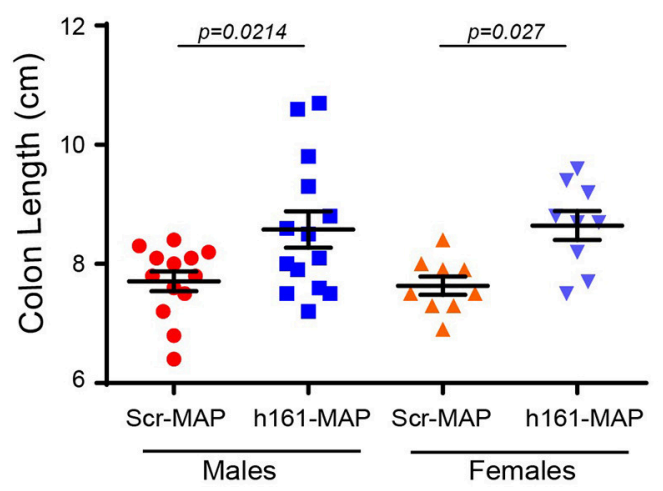

FIGURE 2 | 161-MAP active vaccination prevents damage and extends colon length. Fifteen days after the beginning of DSS-treatment, mice were euthanized and their colons were harvested, formalin-fixed and paraffin-embedded. Colon tissue sections were H\&E stained and analyzed for their histological scores. (A) Representative images and (B) the assessment of the histological score ( $n=6$ in each group). Scale bar is $100 \mu \mathrm{m}$. (C) Representative images of the entire colon removed from male mice, and (D) measurement of the colon lengths ( $n=12$ for the male groups, $n=9$ for the female groups).

and therefore, staining in both cases was strong. However, the immune reactive score (H-score), which takes into account both the intensity of the staining and the number of cells stained with each intensity, allowed us to observe a reduction in EMMPRIN expression in the 161-MAP vaccinated group (Figure 3A), in both male (by 1.6-fold, $p=0.0022,95 \%$ CI $[7.16,25.43]$ ) and female mice (by 1.8 -fold, $p<0.0001,95 \%$ CI $[19.9,30.45]$ ). Moreover, reduced EMMPRIN expression is clearly visualized in the crypts' epithelial cells from 161-MAP vaccinated mice, whereas an increase in infiltrating macrophages is clearly seen in the LP (Figure 3B). Lastly, determination of EMMPRIN levels in the colon lysates (Figure 3C) showed a marked reduction in the 161-MAP vaccinated male (by 1.45 -fold, $p=0.0248,95 \%$ CI [140, $1878]$ ) and female (by 1.36 -fold, $p=0.03,95 \%$ CI $[89.1,1596]$ ) mice, whereas EMMPRIN levels in the circulation were markedly reduced only in the male group (by 1.3 -fold, $p=0.037$ ).

Angiogenesis was evaluated by the microvessel density that was assessed by CD31 staining. In the control Scr-MAP vaccinated groups, blood vessels were abundant in the muscularis mucosa, and infiltrated into the LP (Figure 4A). In contrast, both male and female 161-MAP vaccinated mice exhibited reduced amount of blood vessels in both the muscularis mucosa and in the LP (by 1.6-fold, $p<0.001,95 \% \mathrm{CI}$ for male $4.5,8.9$ ], and for females $[5.9,9.2]$. As expected, the reduction in EMMPRIN levels, a known inducer of VEGF and MMPs expression, led to a similar reduction in local VEGF levels in both male and female mice (by 3 - and 5-fold, respectively, $p<0.05,95 \% \mathrm{CI}$ for male $[0.08,2.5]$ and for female $[0.2,1.35])$, whereas in the serum VEGF was hardly detected in both genders for both 161-Scr-MAP and 161-MAP vaccinated mice (Figure 4B). However, colon MMP-9 levels were reduced only in the 161-MAP male vaccinated group (by 3 -fold, $p=0.0383,95 \% \mathrm{CI}[6.3,197.6])$, but not in the female vaccinated group. Although the local reduction of EMMPRIN levels was reflected in the serum, MMP-9 serum levels and the lack of VEGF serum levels were unchanged by the 161-MAP vaccination.

To demonstrate the involvement of EMMPRIN in the angiogenic potential, we subjected the extracted proteins to bEND3 endothelial scratch assay. The ability of the lysates to trigger wound healing, that requires endothelial cell proliferation 
A
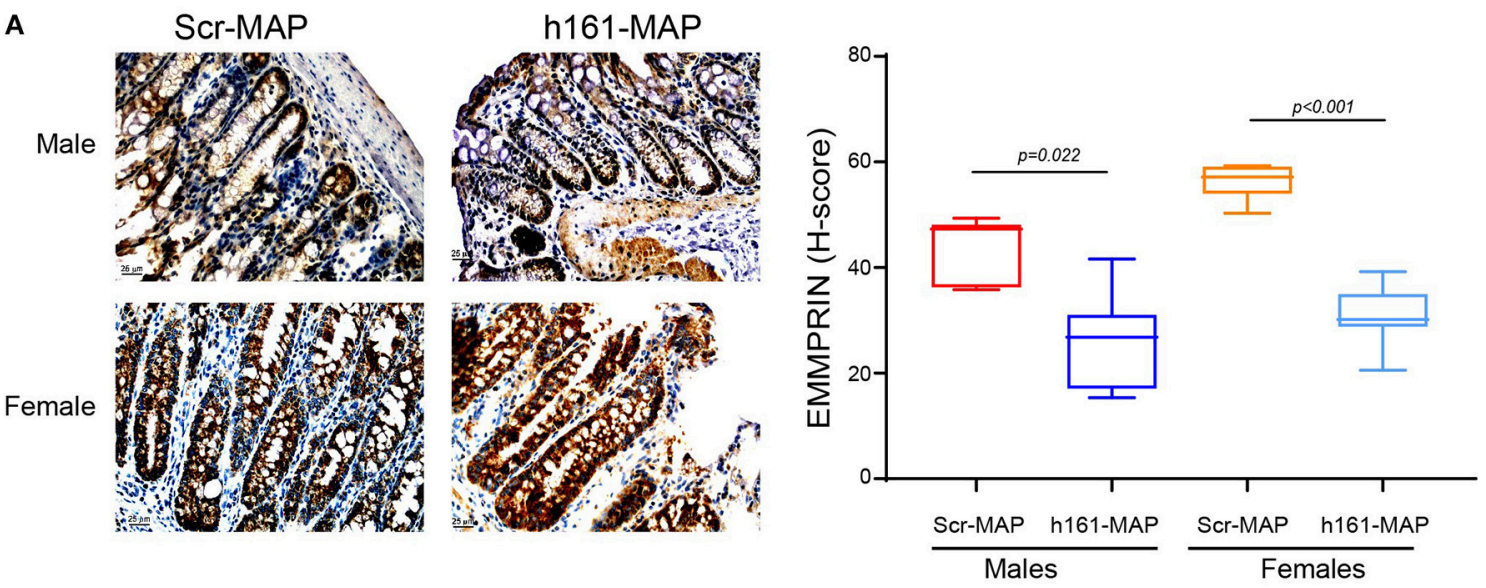

B
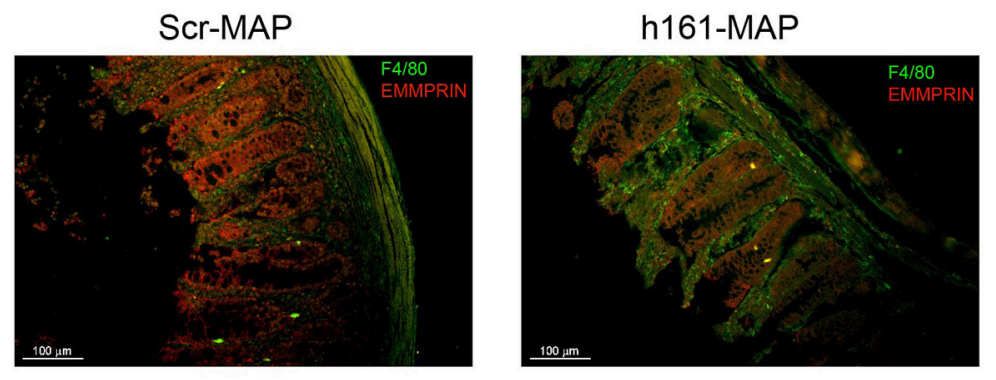

C

\section{Colon}

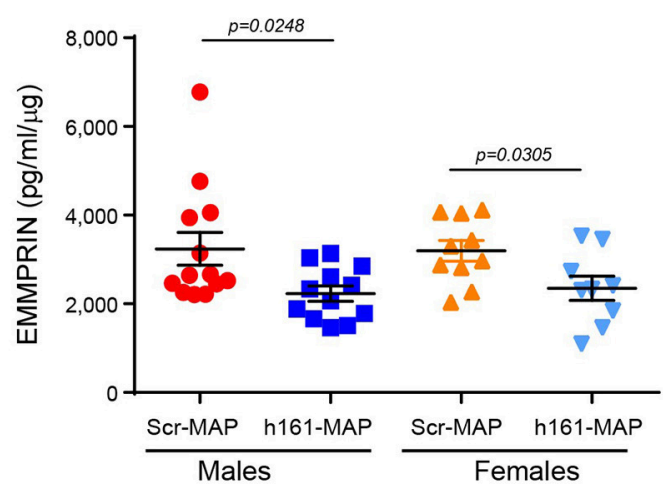

\section{Serum}

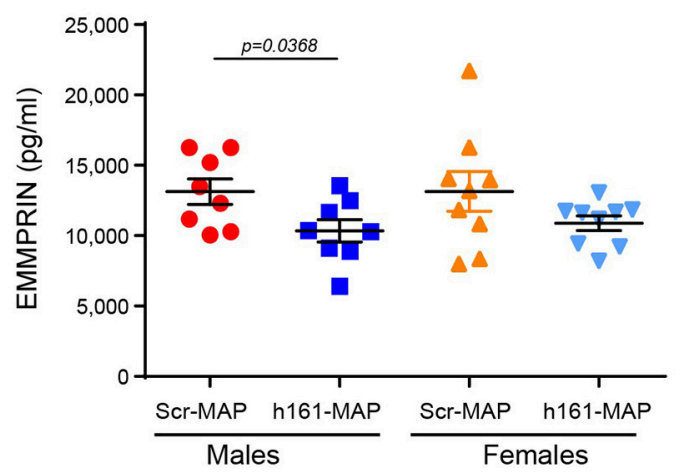

FIGURE 3 | 161-MAP active vaccination reduces EMMPRIN expression in the colon. (A) Representative images of colon sections stained for EMMPRIN and their quantification using the $\mathrm{H}$-score ( $n=5$ per group). Scale bar is $25 \mu \mathrm{m}$ (B) Representative image of fluorescently labeled EMMPRIN (red) and macrophages (green). Scale bar is $100 \mu \mathrm{m}$. (C) Determination of EMMPRIN concentrations in colon lysates $(n=9-10$ per group), and in serum samples $(n=8-9$ per group) by ELISA.

and migration, was assessed by measuring the area to which bEND3 cells had migrated, and the involvement of EMMPRIN was demonstrated by the neutralizing activity of the antiEMMPRIN antibody (161-pAb). The baseline values of bEND3 cell migration, without addition of any protein extract, were similar and not different from the control Scr-MAP group (mean migration area 306,085 $\pm 20,493 \mathrm{~mm}$ ). Relative to lysates obtained from the male Scr-MAP control group, the 161-MAP vaccinated male group reduced the migration of endothelial cells (by 1.42 -fold, $p<0.047,95 \%$ CI [23,426, 157,945], Figure 4C), and the addition of the antibody to male Scr-MAP lysates had a similar effect $(p<0.045)$. Likewise, the 161-MAP vaccination reduced migration in the female mice (1.6-fold, $p<0.019$, 95\% CI $[133,284,474,369])$ (data not shown). However, addition of the antibody to the 161-MAP colon lysates in both male and female mice did not show any additional effect (Figure 4C), suggesting that the vaccination already neutralized EMMPRIN in these lysates. 
A
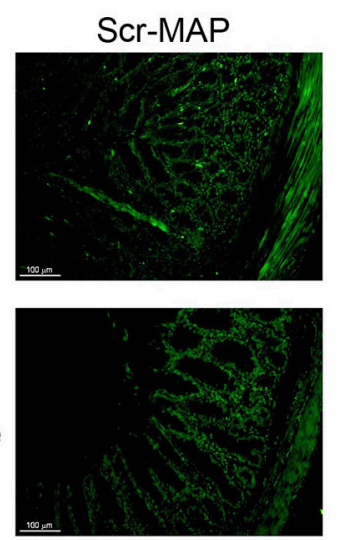
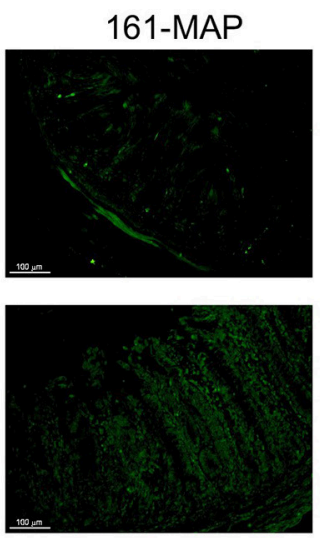

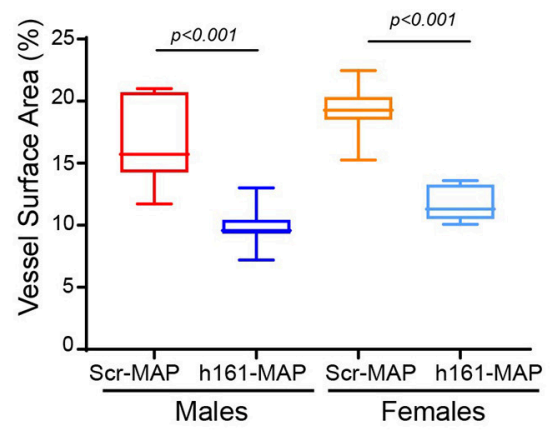

Colon

B

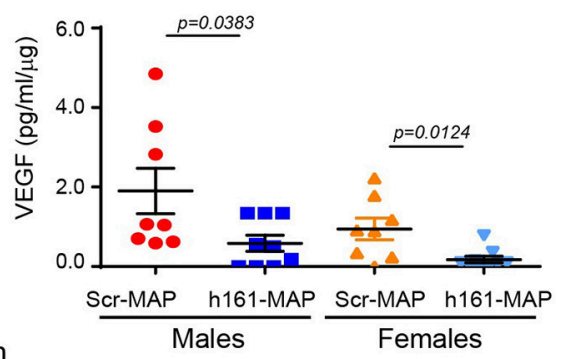

Serum

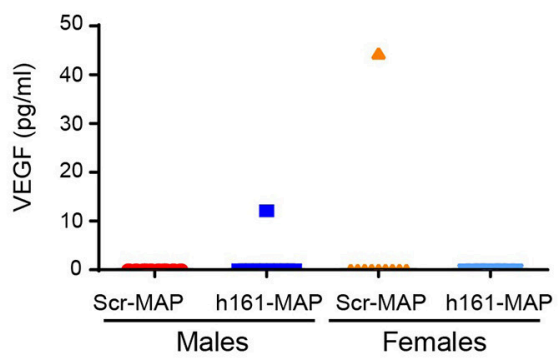

C

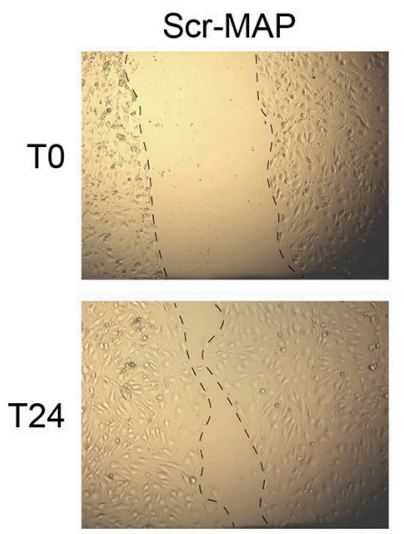

161-MAP
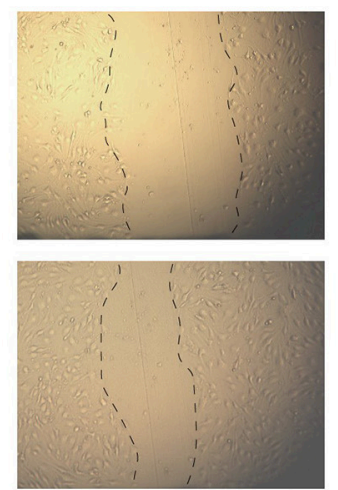
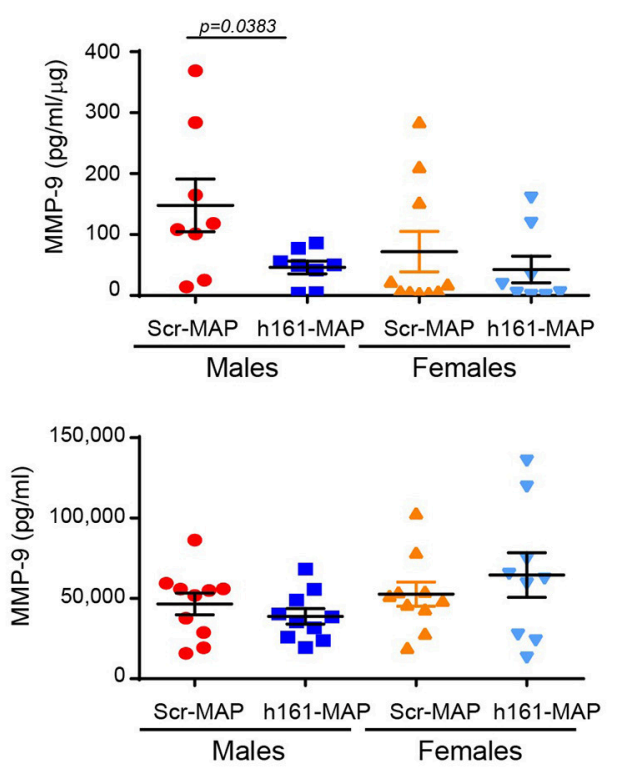

FIGURE 4 | 161-MAP active vaccination reduces angiogenesis. (A) Colon sections were stained for CD31 and the vessel surface area was calculated $(n=4$ per group). Scale bar is $100 \mu \mathrm{m}$. (B) Concentrations of VEGF and MMP-9 were determined in serum sample by ELISA, and in the colon lysates, normalized to the total protein amounts ( $n=9$ per group). (C) Wound scratch assay: colon lysates (25 $\mu \mathrm{g}$ of total protein) were diluted (1:4) and applied onto a confluent layer of the mouse bEND3 endothelial cells ( $10^{5}$ cells/ 96 -plate well) that was scratched with a toothpick. Images were acquired at the beginning of the experiment (TO) and at the end after 24h (T24). The migration area was calculated by subtracting the area of the wound at T24, after endothelial cell migrated and partially closed the wound, from the area of the wound at TO. An EMMPRIN specific blocking antibody (161-pAb) was added to some of the wells as indicated. $(n=9-10$ for the male mice, $n=8$ for the female mice). Magnification is $x 4$. 


\section{1-MAP Vaccination Changes Immune Cell Infiltration, the Microenvironment, and Cell Viability}

As DSS-induced colitis is an inflammatory disease, and the vaccination process is likely to stimulate immune cells, we next stained colon sections for the presence of $\mathrm{CD} 8^{+} \mathrm{T}$ cells, macrophages and neutrophils. As expected, infiltration of $\mathrm{CD} 8^{+}$ $T$ cells, reflecting the stimulation of the adaptive immune system, was increased in the 161-MAP vaccinated mice in comparison to the control mice (by 3.3-fold, $p=0.012$ for the males, $95 \%$ CI $[0.3,0.76]$, by 2.5 -fold, $p=0.0071$ for the females, $95 \%$ CI [0.14, 0.59] Figure 5A). Likewise, infiltration of macrophages was also increased (by 2.2-fold, $p=0.0016$ for the males, $95 \%$ CI $[0.41,1.4]$, by 2.8 -fold, $p<0.001$ for the females, $95 \%$ CI $[1.2,2.1]$, Figure 5B). In contrast, neutrophil infiltration, that is characteristic of the innate immunity in acute inflammation, remained unchanged (data not shown).

To learn about the mode of activation of these cells, we next evaluated cytokine concentrations in serum samples and locally in the colon lysates. Concentrations of IL- $1 \beta, \mathrm{TNF} \alpha$, and IL-10 in the serum and the colon were not different between the control and the 161-MAP vaccinated mice, in both males and females (Figures 5C,D), suggesting that the pro-inflammatory process was no longer active at this late stage. In contrast, levels of TGF $\beta$ were reduced in colon lysates of 161-MAP vaccinated male mice in comparison to the Scr-MAP vaccinated mice (by 3 -fold, $p=$ $0.03,95 \%$ CI $[0.4,1.7])$, whereas in female mice this trend (2fold difference) did not reach significance. In the serum samples, TGF $\beta$ was reduced in both male and female 161-MAP vaccinated mice relative to their respective controls (by 1.4-fold and 1.5fold, $p<0.05,95 \%$ CI for male $[4,357,42,198]$ and for female $[3,703,42,412])$. The high levels of TGF $\beta$ in the control Scr-MAP vaccinated mice together with no change in the low levels of the pro-inflammatory cytokines suggest that by day 15 , the proinflammatory response was already replaced with a regeneration program. The relative reduction in those TGF $\beta$ levels in 161MAP vaccinated mice suggests that a moderate repair program was in place.

Staining the colon sections for Ki-67 revealed that proliferation in the 161-MAP vaccinated groups was reduced relative to the Scr-MAP controls (by 3.7-fold, $p<0.001$ for males $95 \%$ CI $[0.016,0.03]$, and by 2.4 -fold, $p<0.001$ for females, 95\% CI [0.009, 0.015], Figure 6A). In contrast, the number of apoptotic cells, as assessed by the TUNEL assay, was increased in the 161-MAP vaccinated mice relative to the Scr-MAP vaccinated controls (by 2.5 -fold, $p=0.0005$ for males, $95 \%$ CI [176.6, 544.6], by 2.3 -fold, $p=0.0001$ for females, $95 \%$ CI [191.2, 507.3], Figure 6B).

\section{DISCUSSION}

The mechanisms that drive UC and its analogous model of DSS-induced colitis are not fully understood, although increased ROS and pro-inflammatory cytokines, pathological angiogenesis, as well as the composition of the microbiota in the gut have been implicated $(4,6)$. In many of the treated patients, the drugs currently in use do not exert sufficient beneficial effects, suggesting that additional pathological mechanisms, that could potentially be targeted, are at play. Here we demonstrate that by selectively targeting EMMPRIN, a multifunctional protein that is primarily involved in angiogenesis, we reduced angiogenesis and ameliorated clinical manifestations of the DSS-induced colitis model, including weight loss and disease severity, pointing to the central role that angiogenesis plays in this model.

EMMPRIN is highly expressed in the colon, especially in the crypt base columnar cells. As the chaperon of the monocarboxylate transporter family (particularly of MCT- 1 and MCT-4) it has an important function in the transport of monocarboxylate anions, such as lactate, pyruvate, ketone bodies and the short-chain fatty acids acetate, propionate and butyrate (20), all of which are particularly important in intestinal function. Additionally, EMMPRIN may help in recruiting leukocytes to the inflamed site, and support their adhesion to endothelial cells. Despite its important role, this is the first demonstration of the involvement of EMMPRIN in colitis, to the best of our knowledge. We show here that targeting EMMPRIN reduces angiogenesis, but the reduction in EMMPRIN expression in the 161-MAP vaccinated mice suggests that other functions of EMMPRIN may also be affected. These aspects deserve additional exploration that is outside the scope of the current work.

The vaccination reduced EMMPRIN expression and led to reduced microvessel density and angiogenesis. Angiogenesis is a necessary process, as it promotes and sustains inflammation by supplying nutrients, allowing increased leukocyte influx and promoting endothelial cell local production of chemokines, cytokines, and MMPs. In fact, many mediators exhibit a dual role as pro-angiogenic and pro-inflammatory, linking the two processes. For example, VEGF, which is a potent pro-angiogenic factor as well as a chemoattractant for macrophages, has been shown to increase mucosal angiogenesis, promote leukocyte adhesion and worsen the clinical outcome in both IBD patients and in a DSS-induced colitis model (7). Pathogenic angiogenesis and elevated levels of both VEGF and MMP-9 were demonstrated in different models of UC, including a DSS-induced colitis model $(7,21)$. Furthermore, VEGF has been implicated in increasing vascular permeability, which in the context of colitis may have an additional effect by allowing bacteria to invade into the LP, thus enhancing the inflammatory process $(10,22)$.

In the past, attempts have been made to target VEGF or its receptor VEGFR2. For example, targeting VEGFR2 with the monoclonal DC101 antibody in a DSS-induced colitis model did not inhibit angiogenesis or improve disease severity, probably due to VEGF-independent compensatory pathways that maintained downstream signaling events (23). The authors suggested that this monotherapy might have had better effects if used in combination with another monoclonal antibody that targeted another angiogenic mediator. This may have been the case in our EMMPRIN vaccination, as EMMPRIN is a mediator upstream of VEGF that also induces MMP-9. Indeed, we show a reduction (at least in the male groups) of both VEGF and MMP-9, two potent pro-angiogenic mediators. Thus, in contrast to the previous study, we succeeded in improving disease 
A CD8+T cells

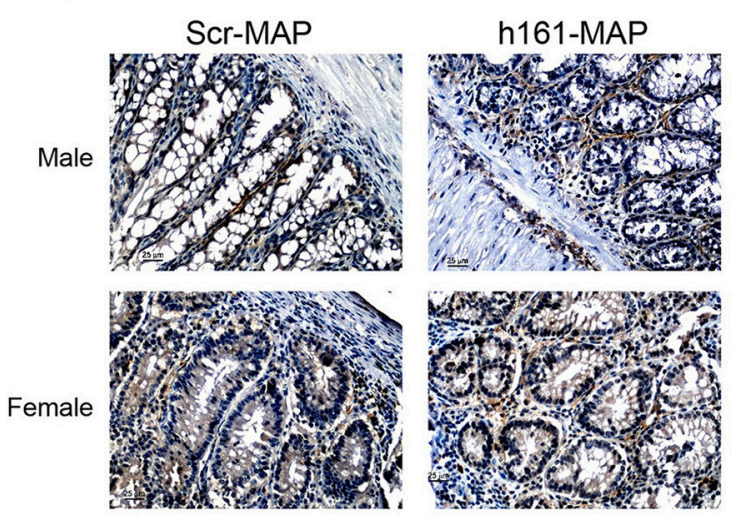

B Macrophages

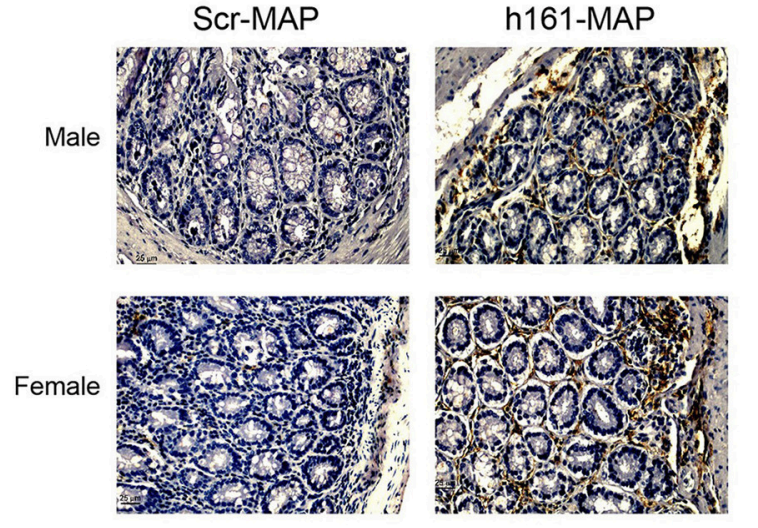

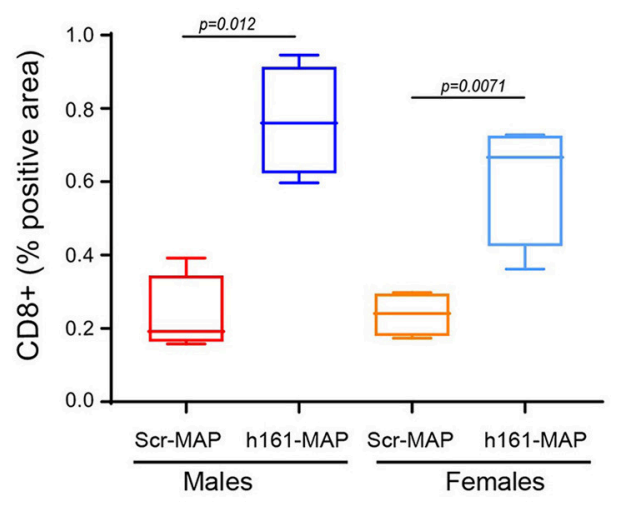

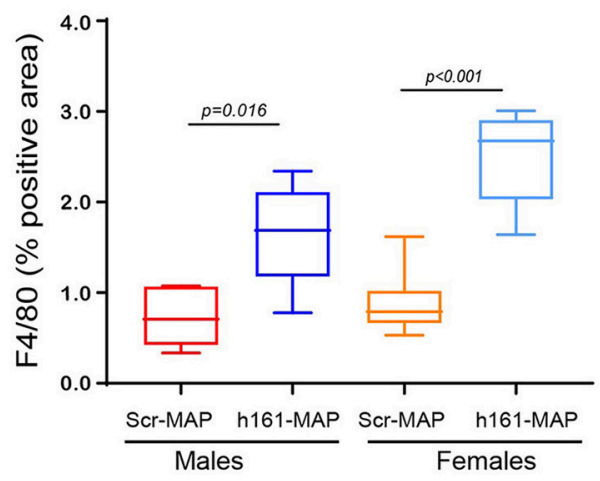

C Colon

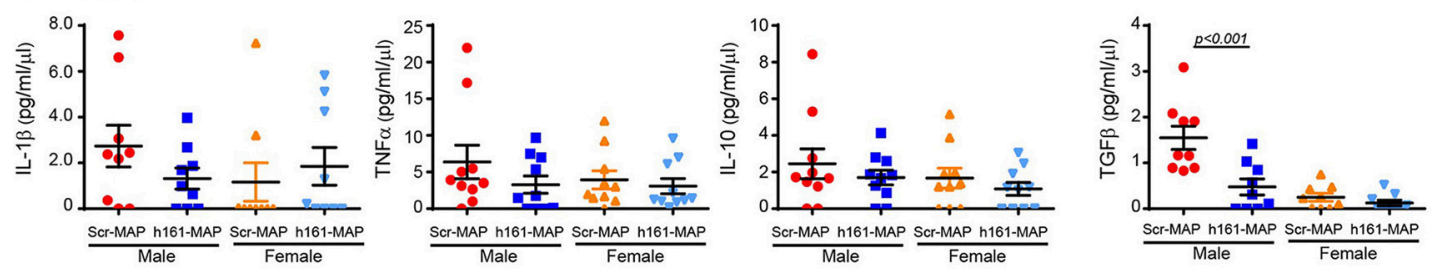

D Serum
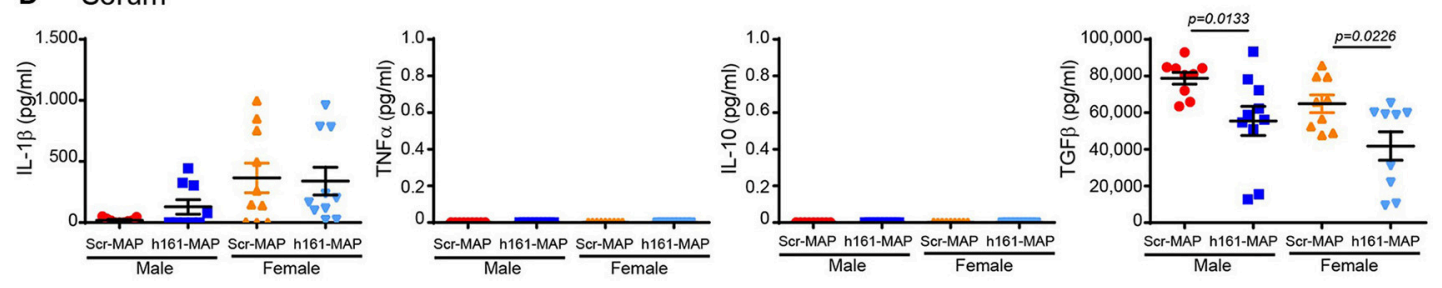

FIGURE 5 | 161-MAP active vaccination increases infiltration of CD8 ${ }^{+} T$ cells and macrophages, and reduces TGF $\beta$ concentrations. Colon tissue sections were stained for (A) $\mathrm{CD}^{+}$or (B) for F4/80, and analyzed for their distribution ( $n=4-5$ in each group). Scale bar is $25 \mu \mathrm{m}$. (C) Proteins were extracted from colon segments and the concentrations of IL-1 1 , TNF $\alpha$, IL-10 and TGF $\beta$ were determined in the lysates by ELISA and normalized to the total protein ( $n=9-10)$. (D) Concentrations of the same cytokines were determined in the serum samples $(n=9-10)$.

severity and in reducing angiogenesis, while demonstrating again the importance of VEGF in the DSS-induced colitis model.
Since inflammation and angiogenesis are interconnected processes, we expected that targeting angiogenesis through EMMPRIN vaccination would also reduce inflammation. For 


\section{A}
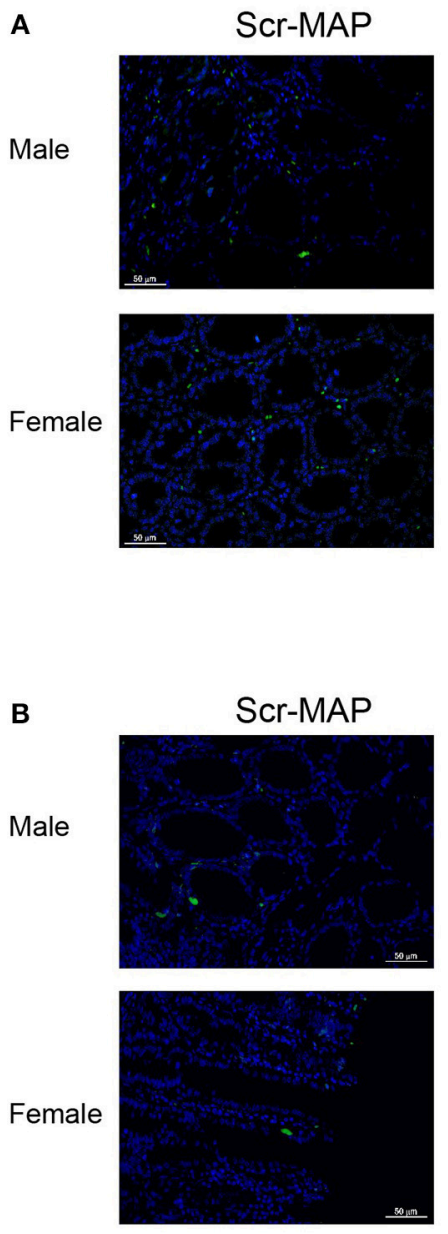

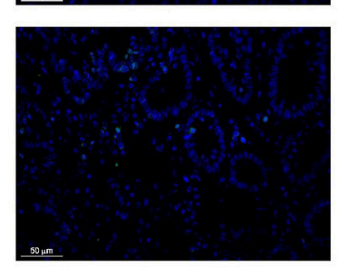

\section{1-MAP}
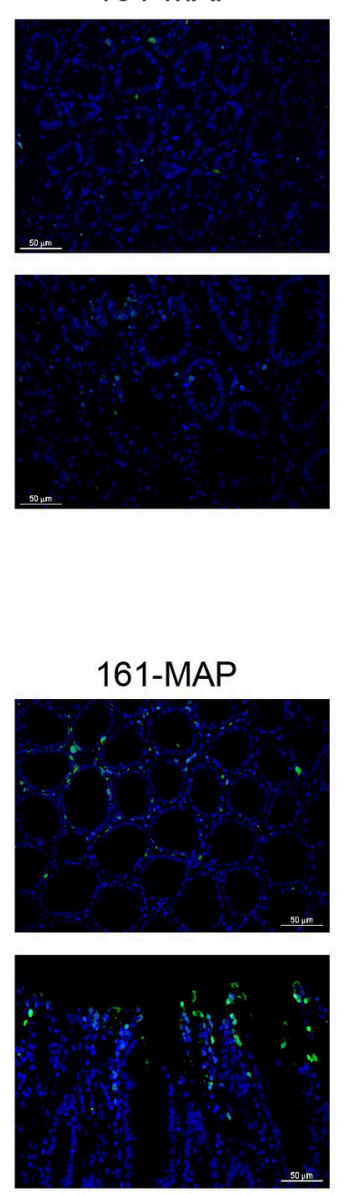
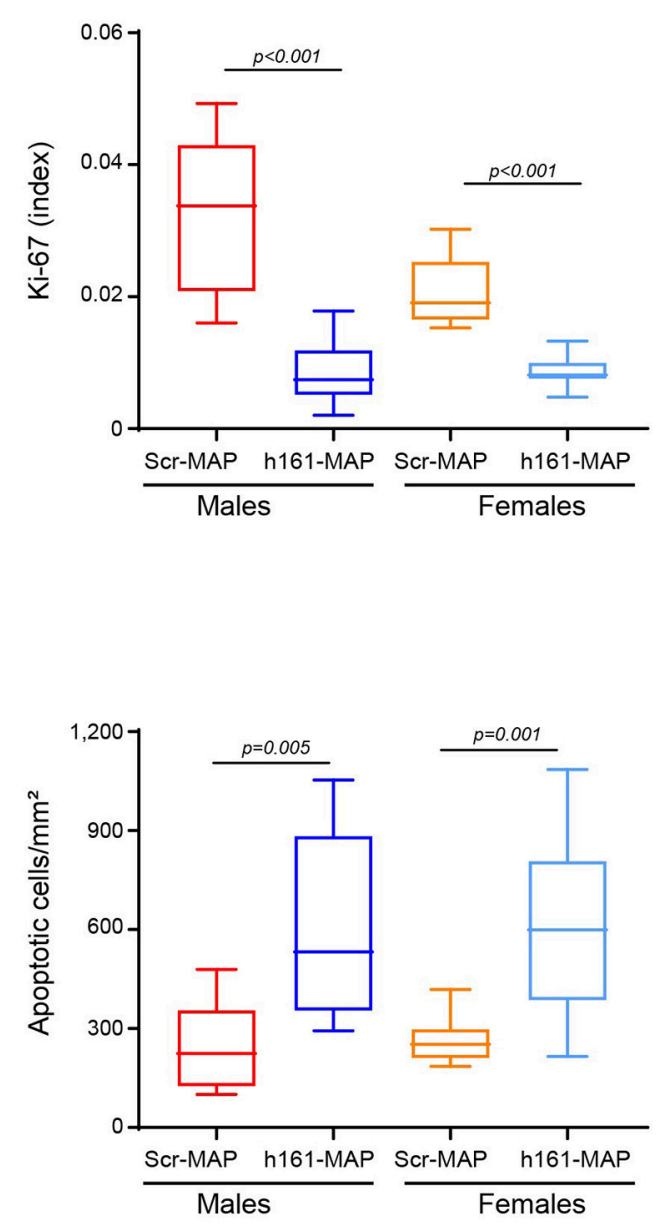

FIGURE 6 | 161-MAP active vaccination reduces proliferation and enhances apoptosis. Colon tissue sections were stained for Ki-67 or DNA strand breaks (TUNEL assay). (A) Representative images of Ki-67 staining (scale bar is $50 \mu \mathrm{m}$ ) and their quantitation (Green, proliferating cells; blue, DAPI staining of nuclei; white, merged co-localization, $n=3-4$ in each group). (B) Representative images of the TUNEL assay and their quantitation. (Green, apoptotic cells; blue, DAPI staining of nuclei; white, merged co-localization, $n=3-4$ in each group). Scale bar is $50 \mu \mathrm{m}$.

example, reduction of VEGF using anti-VEGF antibody has reduced vascular permeability and influx of immune cells into the colon in an experimental colitis model (22). EMMPRIN itself has a role in recruiting leukocytes, and therefore, targeting it was expected to reduce immune infiltrate. However, we show that 15 days after the onset of inflammation by the DSS, more macrophages and $\mathrm{CD}^{+} \mathrm{T}$ cells were present in the LP, and colonic levels of pro-inflammatory cytokines, such as TNF $\alpha$ and IL-1 $\beta$, were unchanged by the vaccination. We suggest that these results can be explained by the duration of the model. Most studies, with or without interventions, follow a design where DSS is administered for 5-7 consecutive days and then the mice are immediately euthanized without a DSS-free period that allows regeneration and repair. Thus, the status of immune activation is measured at the peak of the innate inflammatory response. In our experimental design, the mice are allowed to drink DSSfree water after 5 days of exposure, giving them the chance to repair intestinal damage. In this kinetics, disease is most severe around day 9, 4 days after DSS is no longer administered. It might be argued that since we stimulated the adaptive immune system prior to DSS administration, it is possible that the innate and adaptive immune responses occur simultaneously, prolonging the time of maximal damage. However, since the irritation to the intestine was stopped after 5 days by supplying DSS-free drinking water, the mice have entered into a repair or healing stage. Thus, the intestinal milieu was probably reflecting a resolution state, rather than a pro-inflammatory response with high cytokine concentrations, even if the inflammatory cells were still physically present in the microenvironment. Supporting our premise are studies of DSS-induced colonic tissue that show reduced cytokine production at the mRNA or protein levels in the resolution phase compared to the acute phase of inflammation (24-26).

Several evidences support our conclusion that the immune response is at the repair stage. First, the low number of 
infiltrating neutrophils in the colon samples did not change upon vaccination, suggesting that the system was no longer in acute inflammation. In contrast, during the acute phase of DSS-induced colitis model the innate immune cells, especially neutrophils and macrophages, massively infiltrate the LP, and elevated levels of the pro-inflammatory cytokines they secrete, such as TNF $\alpha$, IL-1 $\beta$, and IL-17 are observed (27). However, upon removal of DSS, the acute response gradually changes into a chronic response, pro-inflammatory cytokines are decreased, and Th2 cytokine levels are increased $(1,2)$. In particular, IL-4 and TGF $\beta$ have been shown to be critical for regeneration of intestinal epithelial cells $(28,29)$. Comparing vaccinated and control mice, we found no change in the amount of infiltrating neutrophils, but $\mathrm{CD}^{+} \mathrm{T}$ cells and macrophages were increased, suggesting that the adaptive immune response, rather than the innate immunity, was specifically increased in the 161-MAP vaccinated mice. The levels of TNF $\alpha$, IL-1 $\beta$, and IL-10 were low and showed no difference between the groups, whereas TGF $\beta$ levels were reduced compared to the Scr-MAP vaccinated control mice. This is consistent with the importance of this cytokine in tissue regeneration, and suggests that the damage in the vaccinated mice during the early stages was relatively reduced, leading to a reduced need for regeneration. Furthermore, turnover of the surface epithelium in the colon takes about 5-8 days. Although signs of inflammation are clearly visible in the vaccinated mice, they also demonstrate restoration of the crypts and epithelium, suggesting reduced inflammation and damage. Alternatively, we surmise that the 161-MAP vaccination triggered an early EMMPRIN-specific pro-inflammatory response, allowing for DSS-damaged epithelial cells that express EMMPRIN to be cleared faster, and helping to promote a rapid regeneration, which was reflected by the reduced loss of weight and disease activity scores.

DSS is believed to directly kill intestinal epithelial cells, cause barrier dysfunction and induce innate immunity, all leading to enhanced epithelial injury during acute inflammation (3, 4 , 30). Studies show that relative to control mice without colitis, DSS-induced colitis increases apoptosis and decreases cell proliferation at the early stages of acute colitis, thus contributing to the barrier dysfunction (31). However, once DSS is removed, the intestine begins to proliferate, in order to regenerate the epithelial layer and restore epithelial barrier function. Increased proliferation underlies an attempt to regenerate the epithelial layer and restore barrier functions $(3,5)$. Thus, we would expect increased proliferation and reduced apoptosis during the regeneration phase in the 161-MAP group. In contrast, we demonstrate reduced proliferation and enhanced apoptosis in the 161-MAP vaccinated mice relative to the control ScrMAP vaccinated mice. We suggest that the high proliferation in the Scr-MAP groups reflects an ongoing regeneration at this time point (10 days after cessation of DSS administration), whereas the relatively reduced proliferation observed in the 161MAP vaccinated groups indicates earlier recovery and a reduced need for regeneration at this time. Likewise, the enhanced apoptosis observed in the 161-MAP vaccinated groups may reflect the death of both epithelial and non-epithelial cells, for example neutrophils, which typically appears at the end of the regeneration phase. As apoptotic neutrophils have been shown to shift macrophage activation toward a healing phenotype (32), and based on the improvement in the DAI and weight loss in the 161-MAP vaccinated groups, we propose that the increase in apoptotic cells may in fact protect the colon and help reduce inflammation.

The protective effects of the 161-MAP vaccination in the DSSinduced colitis model are comparable to our recent results with the same vaccine in implanted and metastatic tumor models, where we used the CT26 colon carcinoma cells among others. We demonstrated there that relative to Scr-MAP vaccinated mice, tumors or metastases were reduced and even eliminated, angiogenesis and its mediators VEGF and MMP-9 were reduced, more $\mathrm{CD}^{+} \mathrm{T}$ cells and macrophages infiltrated the tumors and were engaged in killing tumor cells, TGF $\beta$ was reduced and an increase in a Th1/M1 gene signature was detected (33). However, tumor models represent an ongoing chronic inflammation, with continuous exposure to inflammation-inciting triggers and mediators. In contrast, in our design of the DSS-induced colitis model, we allowed enough time for regeneration following cessation of DSS administration. To delineate the full spectrum of the protective effects of the 161-MAP vaccination in the DSSinduced model, a follow-up study looking at multiple time points during the dynamic healing process should be conducted, and immune cells should be isolated from the LP to phenotype and characterize their exact mode of activation.

Many autoimmune diseases are known for their gender bias, generating our interest to examine this phenomenon in our DSSinduced colitis model. Indeed, we observed that despite the basic similarities in kinetics, the female group generally exhibited a more moderate inflammation, reflected by less severe weight loss and DAI scores. This is in agreement with other studies that found that male mice respond faster and develop a more significant and aggressive colitis relative to female mice when exposed to DSS (3), and that STAT-1 deficiency or IRAK-1 deficiency render male, but not female, mice more resistant to DSS-induced colitis $(34,35)$. However, not all parameters were consistent with this trend, as the histology score and the colon length were similar between males and females. Other studies that showed difference in weight loss but no difference in colon length between the genders attributed these findings to mild colitis being induced $(35,36)$.

In summary, we show that the critical component of angiogenesis can be targeted in DSS-induced colitis, by vaccinating against the pro-angiogenic mediator EMMPRIN protein. This vaccination improved disease severity, reduced angiogenesis and expedited regeneration, although a direct effect on inflammatory cytokines was not observed.

\section{MATERIALS AND METHODS}

\section{Experimental Mouse Model, Vaccination and Disease Activity Index (DAl)}

C57BL/6J OlaHsd male and female mice (8 weeks old, Envigo Laboratories, Jerusalem, Israel), were housed in specific pathogen free (SPF) conditions and kept with a $12 \mathrm{~h}$ light/dark cycle and access to food and water ad libitum. To vaccinate mice we used the synthetic multiple antigenic peptide (MAP) derived 
from the human sequence of the EMMPRIN protein (sequence: GHRWLKGGVVLC, designated h161-MAP), or a peptide with the same amino acids in a scrambled order used as a negative control (sequence: WCRGGGLKMRVH, designated Scr-MAP). Peptides were synthesized by the standard stepwise solidphase procedure using Fmoc chemistry on $\beta$-Ala-Wang resin, conjugating the peptides onto an octa-branched lysine core (Yuan Yu Bio-Teck), and purity was confirmed by HPLC and mass spectroscopy. Using the human sequence, rather than the mouse sequence, reflected the homology between the two sequences, and was shown before to trigger an equally effective EMMPRIN-specific response (33). For the first vaccine injection, the 161-MAP and Scr-MAP (50 $\mu \mathrm{g}$ each) were emulsified in complete Freund's adjuvant (CFA) for the first vaccine injection, and additional three vaccination injections where the same amount of MAPs was emulsified in incomplete Freund's adjuvant (IFA), were administered subcutaneously to each mouse every 7 days. Four days after the last boost injection, colitis was induced with 2\% DSS (MW 36-50 kDa, cat. No. 160110, MP Biomedicals, LLC Solon, $\mathrm{OH}$ ) administered in the drinking water for five consecutive days, after which their drinking water were replaced with regular water, and the mice were left for additional 10 days. At the end of the experiment, mice were euthanized and their colon tissue and serum were harvested for later analyses. Disease activity index (DAI) was calculated as the average of loss of weight, stool consistency and bleeding and evaluated daily for each mouse. Change in weight relative to the weight of each mouse on the first day of DSS administration was given the scores: 0 , if $<1 \%$; $1,1-5 \%, 2,5-10 \%, 3,10-15 \%$;,$>15 \%$; Consistency of the stool was assigned the scores: 0 , normal stool; 2, loose or pasty pellets; 4, diarrhea. Presence of occult blood (measured with Hemooccult, SENSA, Beckman Coulter, Brea, CA) was given the following scores: 0 , normal; 2 , positive occult blood; 4 , rectal bleeding.

\section{Histology and Scoring}

Colon sections were fixed in $4 \%$ formalin and paraffin embedded, and then $4 \mu \mathrm{M}$ sections were stained with hematoxylin and eosin (H\&E). Histological scoring, assessing the severity of the model, was based on four parameters. Epithelial loss was scored as follows: 0 , no epithelial loss; 1 , loss of up to $5 \%$ of the epithelial surface; 2 , loss of $5-10 \%$ of the epithelial surface; 3 , loss of $>10 \%$ of the epithelial surface. Crypt integrity was evaluated as follows: 0 , Intact crypt; 1 , loss of $0-10 \%$ of the crypts; 2 , loss of $10-20 \%$ of the crypts, 3 , loss of $>20 \%$ of the crypts. Inflammatory infiltrate was assigned the following score: 0 , no infiltration; 1, mild leukocyte infiltrate; 2, moderate leukocyte infiltrate; 3 , severe leukocyte infiltrate. Depletion of Goblet cells was estimated as: 0 , no depletion of Goblet cells; 1 , mild depletion of Goblet cells; 2, moderate depletion of Goblet cells; 3, severe depletion of Goblet cells.

\section{Immunohistochemistry, Immunofluorescence, and Immune Reactive Score}

Four-micron thick paraffin embedded tissue sections were deparaffinized on a glass slide with xylene substitute K-Clear Plus
(Kaltex) and rehydrated with decreasing ethanol immersions. Antigen retrieval for $\mathrm{Ki}-67$ and F4/80 was performed by microwave heating in citrate buffer $\mathrm{pH}$ 6.0, for CD31 by immersing the slides in $42 \mathrm{mg} / \mathrm{mL}$ Proteinase XXIV solution (Sigma-Aldrich, Rehovot, Israel) for $10 \mathrm{~min}$ at $37^{\circ} \mathrm{C}$, or in 20 $\mathrm{mg} / \mathrm{mL}$ of Proteinase $\mathrm{K}$ in Tris buffer, $\mathrm{pH} 8.0$ for the TUNEL kit. Endogenous peroxidase was quenched in $3 \% \mathrm{H}_{2} \mathrm{O}_{2}$ solution for $10 \mathrm{~min}$, slides were blocked with $5 \%$ BSA and incubated overnight at $4{ }^{\circ} \mathrm{C}$ with the following primary antibodies: rat anti-mouse EMMPRIN (R\&D systems, MAB772, Minneapolis, $\mathrm{MN}$, USA) diluted 1:250; rat monoclonal anti-F4/80 (Abcam, ab6640, Cambridge, UK) diluted 1:200; rabbit polyclonal antiCD8 (Bioss, bs-0648R, Woburn, MA, USA) diluted 1:400. After washing, the antibodies were detected with HRP-Polymer antirabbit (Zytomed, Berlin, Germany) or with the N-Histofine Simple Stain Mouse MAX PO (Rat) (Nichirei Bioscience, Tokyo, Japan) for $1 \mathrm{~h}$ and the DAB substrate Kit (Zytomed systems). All sections were counterstained with hematoxylin (Sigma) and coverslips were applied using Pertex mounting medium (Histolab Products $\mathrm{AB}$ ). For the CD31 and Ki-67, we used the primary antibodies rat monoclonal anti-CD31 (Acris Antibodies, BM4086, Herford, Germany) diluted 1:50 and rabbit monoclonal anti-Ki67 (Abcam, ab16667) diluted 1/140. Secondary antibodies were donkey Alexa Fluor 488-conjugated anti-rat IgG (Abcam, ab150153), or donkey Alexa Fluor 488-conjugated anti-rabbit (Abcam, ab150061), respectively, diluted 1:500. Nuclei were stained with $300 \mathrm{nM}$ DAPI (MP Biochemicals, LLC Solon, OH) and coverslips were applied using the fluorescent mounting medium (Agilent Dako, Carpinteria, CA). The TUNEL staining was performed using the in situ death detection kit POD (Roche Life Science, Mannheim, Germany) according to manufacturer's instructions. All sections were viewed under the bright field trinocular microscope (Olympus BX-60, Tokyo, Japan) and images were acquired with the MS60 camera and the MShot Image Analysis System V1 (MSHOT, Guangzhou Micro-shot Technology Co., Guangzhou, China). Vessel densities were assessed in CD31 stained sections by using a Weibel grid to calculate vessel surface area (37), and the fraction of $\mathrm{Ki}$ 67-positive tumor cells was calculated by the digital image analysis web application ImageJS (38). EMMPRIN expression was assessed using the modified $\mathrm{H}$-score, which assigns an immune reactive score on a continuous scale of $0-300$, based on the percentage of positive cells expressing the protein at different intensities. Staining was divided into three categories: 1 for "light staining," 2 for "intermediate staining," and 3 for "strong staining." The percentage of positive cells was determined according to the positive surface area of cells measured with ImagePro plus 4.5 software, and the score was calculated using the formula: $1 \times(\% 1$ positive cells $)+2 \times(\% 2$ positive cells $)+3$ $\times(\% 3$ positive cells $)$.

\section{Sandwich ELISA}

The mouse cytokines were determined using ELISA kits (R\&D systems,) according to the manufacturer's instructions. Serum samples were diluted 1:4 (for IL-1 $\beta$, IL-10, and TNF) or 1:100 (for TGF $\beta$, MMP-9, and VEGF), and tissue lysate samples were normalized to the total protein. Serum EMMPRIN 
concentrations were measured with an ELISA kit (Abcam, $\mathrm{ab} 215405)$ at a dilution of 1:200, according to the manufacturer's instructions, or normalized to total protein in tissue lysates.

\section{In vitro Wound Scratch Assay}

In vitro wound scratch assay was performed as described before (17), with the mouse bEND3 endothelial cell monolayers $\left(10^{5}\right.$ cells) seeded in 96-well dishes and incubated with $25 \mathrm{mg}$ of total protein extracted from colon samples of the control or 161-MAP vaccinated mice groups. To demonstrate EMMPRIN involvement, we added the rabbit anti-mouse EMMPRIN polyclonal antibody (161-pAb, $2 \mathrm{ng} / \mathrm{ml})$ that we previously produced (19), to some of the wells. Images of the field of injury were acquired at the beginning of the experiment (T0) and after $24 \mathrm{~h}$ (T24) using the ImagePro plus 4.5 software (Media Cybernetics, Inc., Rockville, MD, USA), and the wound area was measured at both times. The migration area, reflecting the area to which endothelial cells migrated in order to close the wound, was calculated by the subtraction of the area at T24 from the area at T0.

\section{Statistical Analyses}

All values are presented as means $\pm \mathrm{SE}$ and all comparisons are presented with the $95 \%$ CI for the difference between the means. Significance between two groups was determined using the twotailed unpaired $t$-test. Differences between experimental groups accounting for time and treatment were analyzed using twoway analysis of variance (ANOVA) and the post hoc Bonferroni's multiple comparison test. $P$-values exceeding 0.05 were not considered significant.

\section{REFERENCES}

1. Eichele DD, Kharbanda KK. Dextran sodium sulfate colitis murine model: an indispensable tool for advancing our understanding of inflammatory bowel diseases pathogenesis. World J Gastroenterol. (2017) 23:6016-29. doi: 10.3748/wjg.v23.i33.6016

2. Ma J, Yin G, Lu Z, Xie P, Zhou H, Liu J, et al. Casticin prevents DSS induced ulcerative colitis in mice through inhibitions of NF-kappaB pathway and ROS signaling. Phytother Res. (2018) 32:1770-83. doi: 10.1002/ptr.6108

3. Chassaing B, Aitken JD, Malleshappa M, Vijay-Kumar M. Dextran Sulfate Sodium (DSS)-induced colitis in mice. Curr Protoc Immunol. (2014) 104:15.25.1-14. doi: 10.1002/0471142735.im1525s104

4. Perse M, Cerar A. Dextran sodium sulphate colitis mouse model : traps and tricks. J Biomed Biotechnol. (2012) 2012:718617. doi: 10.1155/2012/718617

5. Rose WA, Sakamoto K, Leifer CA. Multifunctional role of dextran sulfate sodium for in vivo modeling of intestinal diseases. BMC Immunol. (2012) 13:4-6. doi: 10.1186/1471-2172-13-41

6. Cromer WE, Mathis JM, Granger DN, Chaitanya G V, Alexander JS. Role of the endothelium in inflammatory bowel diseases. World J Gastroenterol. (2011) 17:578-93. doi: 10.3748/wjg.v17.i5.578

7. Scaldaferri F, Vetrano S, Sans M, Arena V, Straface G, Stigliano E, et al. VEGF-A links angiogenesis and inflammation in inflammatory bowel disease pathogenesis. Gastroenterology (2009) 136:585-95.e5. doi: 10.1053/j.gastro.2008.09.064

8. Alkim C, Alkim H, Koksal AR, Boga S, Sen I. Angiogenesis in inflammatory bowel disease. Int J Inflam. (2015) 2015:970890. doi: 10.1155/2015/970890

9. O'Sullivan S, Gilmer JF, Medina C. Matrix metalloproteinases in inflammatory bowels disease: an update. Meditors of Infalm. (2015) 2015:964131. doi: $10.1155 / 2015 / 964131$

\section{ETHICS STATEMENT}

Mice were cared for in accordance with the procedures outlined in the NIH Guideline for the Care and Use of laboratory Animals, and all experiments were performed under the approved protocol (IL-0350315) issued by the Animal Care and Use Committee of the Technion-Israel Institute of Technology.

\section{AUTHORS CONTRIBUTIONS}

ES performed the animal experiments and carried out all ELISA analyses, as well as wound assays. VB was in charge of the immunohistochemical staining. MAR designed the study, analyzed and interpreted the results, and wrote the manuscript.

\section{FUNDING}

This study was supported by the KAMIN project from the Office of the Chief Scientist in Israel's Ministry of Economy (Grant No. 53642), by the Israel Science Foundation (Grant No. 1392/14), and by the Israel Cancer Association (Grant No. 20180051 made available by the ICA USA Board of Directors).

\section{ACKNOWLEDGMENTS}

This publication was made possible through core services and support provided by Drs. Levin-Ashkenazi and SchlensingerLaufer from the Pre-Clinical Research Authority at the TechnionIsrael Institute for Technology.

10. Deng X, Szabo S, Khomenko T, Tolstanova G, Paunovic B, French $\mathrm{S}$ W, et al. Novel pharmacologic approaches to the prevention and treatment of ulcerative colitis. Curr Pharm Des. (2012) 19:17-28. doi: $10.2174 / 13816128130105$

11. Grass GD, Toole BP. How, with whom and when: an overview of CD147 mediated regulatory networks influencing matrix metalloproteinase activity. Biosci Rep. (2016) 36:e00283. doi: 10.1042/BSR20150256

12. Weidle UH, Scheuer W, Eggle D, Klostermann S, Stockinger H. Cancerrelated issues of CD147. Cancer Genomics Proteomics (2010) 7:157-69.

13. Yurchenko V, Constant S, Eisenmesser E, Bukrinsky M. CyclophilinCD147 interactions: a new target for anti-inflammatory therapeutics. Clin Exp Immunol. (2010) 160:305-17. doi: 10.1111/j.1365-2249.2010.04 115.x

14. Belton RJ, Chen L, Mesquita FS, Nowak RA. Basigin-2 is a cell surface receptor for soluble basigin ligand. J Biol Chem. (2008) 283:17805-14. doi: 10.1074/jbc.M801876200

15. Wang Z, Zhao Z, Jiang T, Chen Y, Huang C. The multistep functions of EMMPRIN / CD147 in the tumor angiogenesis. Cell Mol Med. (2016) 2:1-5. doi: $10.21767 / 2573-5365.100012$

16. Voigt H, Vetter-Kauczok CS, Schrama D, Hofmann UB, Becker JC, Houben R. CD147 impacts angiogenesis and metastasis formation. Cancer Invest. (2009) 27:329-33. doi: 10.1080/07357900802392675

17. Tang Y. Regulation of vascular endothelial growth factor expression by EMMPRIN via the PI3K-Akt signaling pathway. Mol Cancer Res. (2006) 4:371-7. doi: 10.1158/1541-7786.MCR-06-0042

18. Zhou J, Zhu P, Jiang JL, Zhang Q, Wu ZB, Yao XY, et al. Involvement of CD147 in overexpression of MMP-2 and MMP-9 and enhancement of invasive potential of PMA-differentiated THP-1. BMC Cell Biol. (2005) 6:2535. doi: 10.1186/1471-2121-6-25 
19. Walter M, Simanovich E, Brod V, Lahat N, Bitterman H, Rahat MA. An epitope-specific novel anti-EMMPRIN polyclonal antibody inhibits tumor progression. Oncoimmunology (2015) 5:e1078056. doi: 10.1080/2162402X.2015.1078056

20. Kirat D, Kondo K, Shimada R, Kato S. Dietary pectin up-regulates monocaboxylate transporter 1 in the rat gastrointestinal tract. Exp Physiol. (2009) 94:422-433. doi: 10.1113/expphysiol.2009.046797

21. Tolstanova G, Deng X, Khomenko T, Garg P, Paunovic B, Chen L, et al. Role of anti-angiogenic factor endostatin in the pathogenesis of experimental ulcerative colitis. Life Sci. (2011) 88:74-81. doi: 10.1016/j.lfs.2010.10.026

22. Tolstanova G, Khomenko T, Deng X, Chen L, Tarnawski A, Ahluwalia A, et al. Neutralizing anti-vascular endothelial growth factor ( VEGF) antibody reduces severity of experimental ulcerative colitis in rats : direct evidence for the pathogenic role of VEGF. Rev Lit Arts Am. (2009) 328:749-57. doi: 10.1124/jpet.108.145128

23. Knod L, Donovan EC, Chernoguz A, Crawford KM, Dusing MR, Frischer JS. Vascular endothelial growth factor receptor-2 inhibition in experimental murine colitis. J Surg Res. (2013) 184:101-7. doi: 10.1016/j.jss.2013.04.026

24. Bento AF, Leite DFP, Marcon R, Claudino RF, Dutra RC, Cola M, et al. Evaluation of chemical mediators and cellular response during acute and chronic gut inflammatory response induced by dextran sodium sulfate in mice. Biochem Pharmacol. (2012) 84:1459-69. doi: 10.1016/j.bcp.2012.09.007

25. Lan A, Blais A, Coelho D, Capron J, Maarouf M, Benamouzig R, et al. Dual effects of a high-protein diet on DSS-treated mice during colitis resolution phase. Am J Physiol Liver Physiol. (2016) 311:G624-33. doi: 10.1152/ajpgi.00433.2015

26. Meers GK, Bohnenberger H, Reichardt HM, Lühder F, Reichardt SD. Impaired resolution of dss-induced colitis in mice lacking the glucocorticoid receptor in myeloid cells. PLoS ONE (2018) 13:e0190846. doi: 10.1371/journal.pone.0190846

27. Zhang X, Wei L, Wang J, Qin Z, Wang J, Lu Y, et al. Suppression colitis and colitis-associated colon cancer by anti-S100a9 antibody in mice. Front Immunol. (2017) 8:1774. doi: 10.3389/fimmu.2017.01774

28. Iizuka M, Konno S. Wound healing of intestinal epithelial cells. World J Gastroenterol. (2011) 17:2161-71. doi: 10.3748/wjg.v17.i17.2161

29. Stevceva L, Pavli P, Husband A, Ramsay A. Dextran sulphate sodium-induced colitis is amelioratedin Interleukin 4 Deficient Mice. Genes Immun. (2001) 2:309-16. doi: 10.1038/sj.gene.6363782

30. Yuan B, Zhou S, Lu Y, Liu J, Jin X, Wan H, et al. Changes in the expression and distribution of claudins, increased epithelial apoptosis, and a mannanbinding lectin-associated immune response lead to barrier dysfunction in dextran sodium sulfate-induced rat colitis. Gut Liver (2015) 9:734-40. doi: $10.5009 /$ gnl14155

31. Araki Y, Mukaisyo K-I, Sugihara H, Fujiyama Y, Hattori T. Increased apoptosis and decreased proliferation of colonic epithelium in dextran sulfate sodium-induced colitis in mice. Oncol Rep. (2010) 24:869-74. doi: 10.3892/or_00000932

32. Greenlee-Wacker MC. Clearance of apoptotic neutrophils and resolution of inflammation. Immunol Rev. (2016) 273:357-70. doi: 10.1111/imr.12453

33. Simanovich E, Brod V, Rahat MM, Drazdov E, Miriam W, Shakya J, et al. Inhibition of tumor growth and metastasis by EMMPRIN Multiple Antigenic Peptide (MAP) vaccination is mediated by immune modulation. Oncoimmunology (2017) 6:e1261778.doi: 10.1080/2162402X.2016.1261778

34. Berglund M, Thomas JA, Fredin MF, Melgar S, Hörnquist EH, Hultgren $\mathrm{OH}$. Gender dependent importance of IRAK-1 in dextran sulfate sodium induced colitis. Cell Immunol. (2009) 259:27-32. doi: 10.1016/j.cellimm.2009.05.009

35. Crnčec I, Modak M, Gordziel C, Svinka J, Scharf I, Moritsch S, et al. STAT1 is a sex-specific tumor suppressor in colitis-associated colorectal cancer. $\mathrm{Mol}$ Oncol. (2018) 12:514-28. doi: 10.1002/1878-0261.12178

36. Wagnerova A, Babickova J, Liptak R, Vlkova B, Celec P, Gardlik R. Sex differences in the effect of resveratrol on DSS-induced colitis in mice. Gastroenterol Res Pract. (2017) 2017:8051870. doi: 10.1155/2017/8051870

37. Weibel ER, Kistler GS, Scherle WF. Practical stereological methods for morphometric cytology. J Cell Biol. (1966) 30:23-38. doi: 10.1083/jcb.30.1.23

38. Almeida JS, Iriabho EE, Gorrepati VL, Wilkinson SR, Grüneberg A, Robbins $\mathrm{DE}$, et al. ImageJS: personalized, participated, pervasive, and reproducible image bioinformatics in the web browser. J Pathol Inf. (2012) 3:25-32. doi: $10.4103 / 2153-3539.98813$

Conflict of Interest Statement: ES and VB declare that the research was conducted in the absence of any commercial or financial relationships that could be construed as a potential conflict of interest. MR is an inventor of a patent (US Grant US9688732B2, EP application EP2833900A4) related to the research described in the manuscript.

Copyright (c) 2018 Simanovich, Brod and Rahat. This is an open-access article distributed under the terms of the Creative Commons Attribution License (CC BY). The use, distribution or reproduction in other forums is permitted, provided the original author(s) and the copyright owner(s) are credited and that the original publication in this journal is cited, in accordance with accepted academic practice. No use, distribution or reproduction is permitted which does not comply with these terms. 